\title{
Non-Schlesinger Isomonodromic Deformations of Fuchsian Systems and Middle Convolution
}

\author{
Yulia BIBILO ${ }^{\dagger}$ and Galina FILIPUK ${ }^{\ddagger}$ \\ $\dagger$ Department of Theory of Information Transmission and Control, Institute for Information \\ Transmission Problems, Russian Academy of Sciences, Bolshoy Karetny per. 19, \\ Moscow, 127994, Russia \\ E-mail: y.bibilo@gmail.com \\ URL: http://www.iitp.ru/en/users/2131.htm \\ $\ddagger$ Faculty of Mathematics, Informatics and Mechanics, University of Warsaw, \\ Banacha 2, Warsaw, 02-097, Poland \\ E-mail: filipuk@mimuw.edu.pl \\ URL: http://www.mimuw.edu.pl/ filipuk/
}

Received November 20, 2014, in final form March 04, 2015; Published online March 13, 2015

http://dx.doi.org/10.3842/SIGMA.2015.023

\begin{abstract}
The paper is devoted to non-Schlesinger isomonodromic deformations for resonant Fuchsian systems. There are very few explicit examples of such deformations in the literature. In this paper we construct a new example of the non-Schlesinger isomonodromic deformation for a resonant Fuchsian system of order 5 by using middle convolution for a resonant Fuchsian system of order 2. Moreover, it is known that middle convolution is an operation that preserves Schlesinger's deformation equations for non-resonant Fuchsian systems. In this paper we show that Bolibruch's non-Schlesinger deformations of resonant Fuchsian systems are, in general, not preserved by middle convolution.
\end{abstract}

Key words: Middle convolution; isomonodromic deformation; non-Schlesinger isomonodromic deformation

2010 Mathematics Subject Classification: 34M56; 44A15

\section{Introduction}

Dettweiler and Reiter's algebraic analogue [14, 15, 16] of Katz' middle convolution [34] is a certain transformation of Fuchsian systems which preserves an index of rigidity. Middle convolution is related to the Euler transformation of solutions of the Fuchsian systems. There have been numerous studies on middle convolution in recent years, including applications to special functions (e.g., [17, 19, 20, 21, 43, 48]), extensions to irregular systems (e.g., [28, 35, 49]) and various others (see, for instance, [2, 11, 12, 13, 14, 18, 26, 39, 40, 41, 47, 50, 51]).

The theory of isomonodromic deformations for Fuchsian systems was developed by L. Schlesinger [44, 45], L. Fuchs [23], R. Garnier [24, 25] (see also [31, 32, 37] and others). Isomonodromy deformations mean that a monodromy group of the Fuchsian system does not depend on the parameters (the location of poles). It is known that in this case the residue matrices satisfy the so-called Schlesinger equation (with certain assumptions to be discussed below). Isomonodromic deformations are, in particular, important in the theory of special functions (especially the Painlevé transcendents), random matrices, the Riemann-Hilbert problems. They are closely connected to the integrability and the Painlevé property.

It is known that there exist non-Schlesinger deformations $[8,9,10]$. The residue matrices of such deformations do not satisfy the Schlesinger equation, but for any values of the parameters the family of Fuchsian systems has the same monodromy representation. The theory of 
non-Schlesinger isomonodromic deformations completes the theory of the isomonodromic deformations.

The study of the resonant Fuchsian systems appeared to be very fruitful in the areas related to the analytic theory of linear differential equations and led to some unexpected results (see [30]). In particular, non-Schlesinger isomonodromic deformations appeared in the following problems. A.A. Bolibruch considered a general isomonodromic differential 1-form in the context of isomonodromic confluence of Fuchsian singularities. The question of isomonodromic confluence was posed by V.I. Arnold and it was included in his list of problems (see [3, Pr. 1984-7, Pr. 1987-12]). In particular, one of the problems was to get a system with irregular singularities as a limit of a Fuchsian isomonodromic family when its singularities coalesce. A.A. Bolibruch proved that it is impossible using the theory of non-Schlesinger isomonodromic deformations. The general isomonodromic differential 1-form was crucial in the solution of this problem. In addition, the existence of examples of non-Schlesinger deformations essentially answers the question posed by Y. Sibuya in [46]. He asked whether it is necessary for the connection matrices of the isomonodromic family with the Fuchsian and irregular singularities to be constant. The answer is that it it not necessary (see Definition 1 below).

There exist some further studies and extensions of the notion of isomonodromic deformations of the Fuchsian systems (see, for instance, $[38,42]$ ). In this paper we deal with the definition of isomonodromic deformations as in Definition 1, Theorems 1 and 2.

In [27] it was shown that the deformation equations for Fuchsian equations are preserved by middle convolution. In particular, the so-called Hitchin systems [29] obtained from the Schlesinger systems are invariant under middle convolution. One of the aims of this paper is to study non-Schlesinger deformations and their behaviour under middle convolution.

Usually it is very difficult to find explicit examples of non-Schlesinger deformations. The first example of the non-Schlesinger isomonodromic deformation which cannot be reduced to the Schlesinger one was constructed by A.A. Bolibruch in [6]. To date, very few examples exist $[4,33,36]$. For instance, to our knowledge, there are no explicit examples of non-Schlesinger deformations for resonant Fuchsian systems with higher order poles in the "non-Schlesinger" part of the isomonodromic differential form, which is defined below. All of the known examples are for Fuchsian systems of orders 2 or 3 . Therefore, a construction of explicit examples for systems of higher order is interesting and new in its own right.

The paper is organized as follows. In the first section we recall the theory of Schlesinger and non-Schlesinger deformations following [9, 10, 31]. Next we briefly summarize the algebraic construction of middle convolution following $[14,15,16]$. We present a new explicit example of the non-Schlesinger isomonodromic deformation of a Fuchsian system of order 5. Finally we present an explicit example which shows that non-Schlesinger isomonodromic deformations are not preserved by middle convolution in general. We also discuss a number of open questions and problems. Some of them are raised by the examples in the current paper.

\section{Isomonodromic deformations}

Let us consider a system of $p$ linear differential equations on the Riemann sphere

$$
\frac{d y}{d z}=\left(\sum_{i=1}^{n} \frac{A_{i}^{0}}{z-a_{i}^{0}}\right) y, \quad \sum_{i=1}^{n} A_{i}^{0}=-A_{n+1}^{0}
$$

with distinct singular points $a_{1}^{0}, \ldots, a_{n}^{0} \in \mathbb{C}, a_{n+1}^{0}=\infty$. Here $y(z) \in \mathbb{C}^{p}$ is unknown. System (1) is a Fuchsian system and its important characteristic is a monodromy group. The monodromy representation is defined as follows. Take an arbitrary non-singular point $z_{0} \in \mathbb{C}$. Let $Y(z)$ be a fundamental matrix solution of system $(1)$ and $\tilde{Y}(z)$ be its analytic continuation along a loop $\gamma$ 
which starts and ends at $z_{0}$. These fundamental matrices are related by $Y(z)=\tilde{Y}(z) G_{\gamma}$, where the matrix $G_{\gamma}$ is constant with respect to $z$ and depends only on the homotopy class $[\gamma]$ of the loop $\gamma$. The mapping $\gamma \rightarrow G_{\gamma}$ defines a representation of the fundamental group into the group of non-degenerate matrices, i.e.,

$$
\chi^{0}: \pi_{1}\left(T^{0}, z_{0}\right) \rightarrow \mathbb{G} \mathbb{L}(p, \mathbb{C}), \quad T^{0}=\overline{\mathbb{C}} \backslash \cup_{i=1}^{n+1}\left\{a_{i}^{0}\right\} .
$$

The generators $\gamma_{1}, \ldots, \gamma_{n}, \gamma_{n+1}$ of the fundamental group (the loops around singularities $\left\{a_{1}^{0}\right.$, $\left.\left.\ldots, a_{n}^{0}, a_{n+1}^{0}\right\}\right)$ satisfy $\gamma_{1} \cdots \gamma_{n} \gamma_{n+1}=e$.

Now consider a family of Fuchsian systems

$$
\frac{d y}{d z}=\left(\sum_{i=1}^{n} \frac{A_{i}(a)}{z-a_{i}}\right) y, \quad \sum_{i=1}^{n} A_{i}(a)=-A_{n+1}(a),
$$

where the location of singularities varies, which is denoted by a parameter $a$, i.e., $a=\left(a_{1}, \ldots, a_{n}\right)$. Moreover, we assume that the initial system (1) is included into the isomonodromic family of (3) with $A_{i}\left(a^{0}\right)=A_{i}^{0}, a^{0}=\left(a_{1}^{0}, \ldots, a_{n}^{0}\right), i=1, \ldots, n$. Similarly to (2) we can define the monodromy representation of system (3) for any $a \in D\left(a^{0}\right) \backslash \cup_{i, j=1, i \neq j}^{n}\left\{a_{i}=a_{j}\right\}$ by

$$
\chi_{a}: \pi_{1}\left(T_{a}, z_{0}\right) \rightarrow \mathbb{G} \mathbb{L}(p, \mathbb{C}), \quad T_{a}=\overline{\mathbb{C}} \backslash \cup_{i=1}^{n+1}\left\{a_{i}\right\} .
$$

Here $D\left(a^{0}\right)$ is a small open disk centered at $a^{0}$.

Definition 1 ([7, 9, 10]). The family of Fuchsian systems (3) is called isomonodromic if the monodromy representation $\chi_{a}$ coincides with the monodromy representation $\chi^{0}$ of the initial system (1) for any $a \in D\left(a^{0}\right) \backslash \cup_{i, j=1, i \neq j}^{n}\left\{a_{i}=a_{j}\right\}$.

Definition 1 means that there exists a fundamental solution $Y(z, a)$ (called an isomonodromic matrix) defining the same monodromy matrices for any $a \in D\left(a^{0}\right) \backslash \cup_{i, j=1, i \neq j}^{n}\left\{a_{i}=a_{j}\right\}$. The isomonodromic family (3) is also called the isomonodromic deformation. There exists a canonical isomorphism between the fundamental groups $\pi_{1}\left(T^{0}, z_{0}\right)$ and $\pi_{1}\left(T_{a}, z_{0}\right)$ (see, for instance, [9]). Therefore, the equality between the monodromy representations $\chi_{a}$ and $\chi^{0}$ is defined correctly.

The following theorem was formulated and proved for $(2 \times 2)$-families of Fuchsian systems in [22]. A.A. Bolibruch generalized it for families of any order.

Theorem 1 ([7, 9, 10]). The family of Fuchsian systems (3) is isomonodromic if and only if there exists a matrix-valued differential 1-form $\omega$ on $\mathbb{C} \times D\left(a^{0}\right) \backslash \cup_{i=1}^{n}\left\{z-a_{i}=0\right\}$ such that

i) $\omega=\sum_{i=1}^{n} \frac{A_{i}(a)}{z-a_{i}} d z$ for any fixed $a \in D\left(a^{0}\right)$;

ii) $d \omega=\omega \wedge \omega$.

Definition 1 was proposed by A.A. Bolibruch (see, for instance, [10]). It extends the notion of the isomonodromic deformation which was used before. The Schlesinger deformations are the most known and well-studied isomonodromic deformations in the literature. They are given by the differential 1-form

$$
\omega_{\text {Schl }}=\sum_{i=1}^{n} \frac{A_{i}(a)}{z-a_{i}} d\left(z-a_{i}\right) .
$$

One can verify directly that condition ii) of Theorem 1 is equivalent to

$$
d A_{i}(a)=-\sum_{j=1, j \neq i}^{n} \frac{\left[A_{i}(a), A_{j}(a)\right]}{a_{i}-a_{j}} d\left(a_{i}-a_{j}\right),
$$


which is called the Schlesinger equation. It is easily seen that the residue matrices satisfy the so-called Schlesinger equations

$$
\frac{\partial A_{i}(a)}{\partial a_{i}}=-\sum_{k \neq i} \frac{\left[A_{i}(a), A_{k}(a)\right]}{a_{i}-a_{k}}, \quad \frac{\partial A_{j}(a)}{\partial a_{i}}=\frac{\left[A_{i}(a), A_{j}(a)\right]}{a_{i}-a_{j}}, \quad j \neq i .
$$

Note that in [29] N. Hitchin derives a new system of equations from the Schlesinger equations (5) for traces of products of matrices and their commutators $\operatorname{tr}\left(\left[A_{i}, A_{j}\right] A_{k}\right)$.

The fundamental matrix $Y_{\text {Schl }}(z, a)$ of the isomonodromic family defined by the differential 1-form (4) satisfies

$$
Y_{\mathrm{Schl}}(\infty, a) \equiv C
$$

where $C$ is a constant non-degenerated matrix. Indeed, the 1-form of the deformation is defined (see [10]) by using the fundamental matrix $Y(z)$ of the family as follows:

$$
\omega=d Y \cdot Y^{-1},
$$

and, hence,

$$
d_{a} Y_{\mathrm{Schl}}(\infty, a) Y_{\mathrm{Schl}}^{-1}(\infty, a)=-\left.\sum_{i=1}^{n} \frac{A_{i}(a)}{z-a_{i}} d\left(a_{i}\right)\right|_{z=\infty} \equiv 0 .
$$

Definition 2. A deformation of the Fuchsian system is called normalized if the choice of the isomonodromic fundamental matrix of the system is fixed by $Y(\infty, a) \equiv C$, where the matrix $C$ is constant and non-degenerate.

Let us return to the definition of the monodromy representation of the Fuchsian system (1). Take another fundamental matrix $X(z)$ of system (1). Two fundamental matrices $Y(z)$ and $X(z)$ are related by $X(z)=Y(z) M$, where $M$ is a constant non-degenerate matrix. The analytic continuation $\tilde{X}(z)$ of the fundamental matrix $X(z)$ along a loop $\gamma$ satisfies $\tilde{X}(z)=X(z) \tilde{G}_{\gamma}$. Then the monodromy matrices $G_{\gamma}$ and $\tilde{G}_{\gamma}$ are related by

$$
\tilde{G}_{\gamma}=M^{-1} G_{\gamma} M
$$

Therefore, the Fuchsian system (1) defines not only a unique monodromy representation $\chi^{0}$ but a conjugacy (by a constant matrix) class of representations.

To summarise, Definition 1 proposed by A.A. Bolibruch is a natural and well-grounded extension of the notion of the normalized isomonodromic deformation since there is no any requirement that the monodromy representation for any value of the parameter is defined with respect to the fundamental matrix fixed by some principle (see [1] for more details).

An arbitrary isomonodromic deformation is not necessarily the Schlesinger deformation (4). Let us consider a family of Fuchsian systems with the fundamental matrix

$$
Y(z, a)=\Gamma(a) Y_{\mathrm{Schl}}(z, a)
$$

where $\Gamma(a)$ is a holomorphically invertible matrix, $\Gamma\left(a_{0}\right)=I$. In this case the differential 1-form $\omega=d Y(z, a) Y^{-1}(z, a)$ is given by

$$
\omega=\sum_{i=1}^{n} \frac{A_{i}^{\prime}(a)}{z-a_{i}} d\left(z-a_{i}\right)+\sum_{k=1}^{n} \gamma_{k}(a) d a_{k}
$$


where the coefficients are given by $[9,10]$

$$
A_{i}^{\prime}(a)=\Gamma(a) A_{i}(a) \Gamma^{-1}(a), \quad \gamma_{k}(a)=\frac{\partial \Gamma(a)}{\partial a_{k}} \Gamma^{-1}(a) .
$$

This isomonodromic deformation is not normalized. It is clear that this deformation is reduced to the Schlesinger deformation by

$$
Y_{\mathrm{Schl}}(z, a)=\Gamma^{-1}(a) Y(z, a) .
$$

Note that one can include initial system (1) into two isomonodromic deformations defined by $\omega_{\text {Schl }}$ and $\omega$.

However, there exist isomonodromic deformations given by differential 1-forms different from (4) and (6). In [10] A.A. Bolibruch gave examples of such deformations and obtained a general form of the isomonodromic deformation.

Definition 3 ([7, 10]). Let $\lambda_{1}^{i}, \ldots, \lambda_{p}^{i}$ be the eigenvalues of the matrix $A_{i}$ of the Fuchsian system (3). A singular point $a_{i}$ is called resonant if there exist at least two distinct eigenvalues of $A_{i}$ such that their difference is a natural number. The number

$$
r_{i}=\max _{k \neq j,\left|\lambda_{k}^{i}-\lambda_{j}^{i}\right| \in \mathbb{N}}\left|\lambda_{k}^{i}-\lambda_{j}^{i}\right|
$$

is called the maximal $i$-resonance of the system.

Theorem 2 ([7, 10]). Any matrix-valued differential 1-form $\omega$ on $\overline{\mathbb{C}} \times D\left(a^{0}\right) \backslash \cup_{i=1}^{n}\left\{z-a_{i}=0\right\}$ which defines the isomonodromic deformation of (3) is given by

$$
\omega=\sum_{i=1}^{n} \frac{A_{i}(a)}{z-a_{i}} d\left(z-a_{i}\right)+\sum_{k=1}^{n} \gamma_{k}(a) d a_{k}+\sum_{l=1}^{n} \sum_{k=1}^{n} \sum_{m=1}^{r_{l}} \frac{\gamma_{m, k, l}(a)}{\left(z-a_{l}\right)^{m}} d a_{k},
$$

where $\gamma_{m, k, l}(a), \gamma_{k}(a)$ are holomorphic in $D\left(a^{0}\right)$ and $r_{l}$ is a maximal l-resonance of system (3) for $a=a^{0}$.

We remark that the last terms may be non-zero only if system (3) has some resonant singularities.

The famous example $[7,8,10]$ of A.A. Bolibruch of the non-Schlesinger normalized isomonodromic deformation is given as follows.

Example 1. The family of Fuchsian systems with four finite singularities

$$
\begin{aligned}
\frac{d y}{d z}= & \left(\left(\begin{array}{cc}
1 & 0 \\
-\frac{2 a}{a^{2}-1} & 0
\end{array}\right) \frac{1}{z+a}+\left(\begin{array}{cc}
0 & -6 a \\
0 & -1
\end{array}\right) \frac{1}{z}\right. \\
& \left.+\left(\begin{array}{cc}
2 & 3+3 a \\
\frac{1}{1+a} & -1
\end{array}\right) \frac{1}{z-1}+\left(\begin{array}{cc}
-3 & 3 a-3 \\
\frac{1}{a-1} & 2
\end{array}\right) \frac{1}{z+1}\right) y
\end{aligned}
$$

is isomonodromic with the differential form $\omega$ given by

$$
\begin{aligned}
\omega= & \left(\begin{array}{cc}
1 & 0 \\
-\frac{2 a}{a^{2}-1} & 0
\end{array}\right) \frac{d(z+a)}{z+a}+\left(\begin{array}{cc}
0 & -6 a \\
0 & -1
\end{array}\right) \frac{d z}{z}+\left(\begin{array}{cc}
0 & 0 \\
\frac{2 a}{a^{2}-1} & 0
\end{array}\right) \frac{d a}{z+a} \\
& +\left(\begin{array}{cc}
2 & 3+3 a \\
\frac{1}{1+a} & -1
\end{array}\right) \frac{d(z-1)}{z-1}+\left(\begin{array}{cc}
-3 & 3 a-3 \\
\frac{1}{a-1} & 2
\end{array}\right) \frac{d(z+1)}{z+1} .
\end{aligned}
$$


The family (7) is isomonodromic by Theorem 1 . Here $a_{1}=-a$ is the resonant singular point (the corresponding residue matrix has eigenvalues 1 and 0 , so the difference is a natural number and the maximal 1-resonance for the singular point $a_{1}=-a$ is equal to 1) and $a_{2}=0$ is also resonant, 2-resonance is equal to 1 . The deformation is normalized (since there is no term of the form $\gamma(a) d a$ for any holomorphic $\gamma(a))$ and it cannot be reduced to any Schlesinger deformation (since there is a term $\left(\begin{array}{cc}0 & 0 \\ \frac{2 a}{a^{2}-1} & 0\end{array}\right) \frac{d a}{z+a}$ and it cannot be removed by any holomorphic transformation $\left.y=\Gamma(a) y_{1}\right)$.

We remark that the singularity $z=-a$ in the example above is apparent. This can be seen by applying a series of transformations to (7). In particular, changing $y=\operatorname{diag}(a+z, 1) y_{1}$ gives a system with a zero residue matrix at $z=-a$ and, by using an additional transformation $y_{1}=\operatorname{diag}(1 / z, 1) y_{2}$ we get a simple system with three singularities and integer coefficients in all residue matrices.

Finally, we remark that in [36] A. Kitaev also considered non-Schlesinger isomonodromic deformations. He considered some analogues of the Schlesinger equation and studied relations between their solutions and solutions of some Schlesinger equation.

\section{Middle convolution}

Middle convolution $m c_{\mu}$ is an operation on tuples of residue matrices of a Fuchsian system introduced by M. Dettweiler and S. Reiter [15, 16]. For a given parameter $\mu \in \mathbb{C}$ one defines residue matrices of dimension $n p \times n p$ which are partitioned into blocks and have only one non-zero block consisting of initial residue matrices and the parameter $\mu$. By finding invariant subspaces and reducing the size of the matrices (if the invariant subspaces are non-empty) one gets a new Fuchsian system with the same singularities but with new residue matrices. Note that the size of the residue matrices of the resulting system depends on the choice of the parameter $\mu$. Middle convolution $m c_{\mu}$ is, in fact, related to the Euler transformation as shown in [16].

Originally N. Katz [34] gave an algorithm to construct all physically rigid irreducible local systems on $\mathbb{C P}^{1} \backslash\{$ finite points\}. M. Dettweiler and S. Reiter [15, 16] introduced a purely algebraic algorithm and showed its equivalence to the algorithm of Katz. Although initially the algorithm was applied to rigid systems, as it is shown in [19, 27], one can prove non-trivial results for non-rigid systems as well.

Now following $[15,16,20,27]$ we briefly summarize the main steps of the algorithm. Let $\mathbf{A}=\left(A_{1}, \ldots, A_{n}\right), A_{k} \in \mathbb{C}^{p \times p}$ be the residue matrices of the Fuchsian system

$$
\frac{d y}{d z}=\sum_{k=1}^{n} \frac{A_{k}}{z-a_{k}} y
$$

with finite singular points $a_{k} \in \mathbb{C}, k=1, \ldots, n$.

For a given parameter $\mu \in \mathbb{C}$ one defines the so-called convolution matrices $\mathbf{B}=c_{\mu}(\mathbf{A})=$ $\left(B_{1}, \ldots, B_{n}\right)$ by

$$
B_{k}=\left(\begin{array}{ccccccc}
0 & \ldots & 0 & 0 & 0 & \ldots & 0 \\
\vdots & & \vdots & \vdots & \vdots & & \vdots \\
A_{1} & \ldots & A_{k-1} & A_{k}+\mu I_{p} & A_{k+1} & \ldots & A_{n} \\
\vdots & & \vdots & \vdots & \vdots & & \vdots \\
0 & \ldots & 0 & 0 & 0 & \ldots & 0
\end{array}\right) \in \mathbb{C}^{n p \times n p}
$$

such that $B_{k}$ is zero outside the $k$-th block row. 
By using the convolution matrices we define a new Fuchsian system of order $n p$ with the same number of singularities as in the original system:

$$
\frac{d y_{1}}{d z}=\sum_{k=1}^{n} \frac{B_{k}}{z-a_{k}} y_{1}
$$

There are the following invariant subspaces (with respect to $B_{k}$ ) of the column vector space $\mathbb{C}^{n p}$ :

$$
\mathcal{L}_{k}=\left(\begin{array}{c}
0 \\
\vdots \\
0 \\
\operatorname{Ker}\left(\mathrm{A}_{\mathrm{k}}\right) \\
0 \\
\vdots \\
0
\end{array}\right)(k \text {-th entry }), \quad k=1, \ldots, n
$$

and

$$
\mathcal{K}=\bigcap_{k=1}^{n} \operatorname{Ker}\left(B_{k}\right)=\operatorname{Ker}\left(B_{1}+\cdots+B_{n}\right)
$$

Next we fix an isomorphism between $\mathbb{C}^{n p} /(\mathcal{K}+\mathcal{L})$, where $\mathcal{L}=\oplus_{k=1}^{n} \mathcal{L}_{k}$, and $\mathbb{C}^{m}$ for some $m$. Note that if there are non-zero invariant subspaces, then $m<n p$.

Definition 4. The matrices $\widetilde{\mathbf{B}}=m c_{\mu}(\mathbf{A}):=\left(\widetilde{B}_{1}, \ldots, \widetilde{B}_{n}\right) \in \mathbb{C}^{m \times m}$, where $\widetilde{B}_{k}$ is induced by the action of $B_{k}$ on $\mathbb{C}^{m} \simeq \mathbb{C}^{n p} /(\mathcal{K}+\mathcal{L})$, are called the additive version of the middle convolution of $\mathbf{A}$ with the parameter $\mu$.

Finally, the resulting Fuchsian system of order $m$ is given by

$$
\frac{d y_{2}}{d z}=\sum_{k=1}^{n} \frac{\widetilde{B}_{k}}{z-a_{k}} y_{2} .
$$

Thus, middle convolution $m c_{\mu}$ is a transformation on tuples of matrices

$$
\left(\left(A_{1}, \ldots, A_{n}\right) \in\left(\mathbb{C}^{p \times p}\right)^{n}\right) \rightarrow m c_{\mu}\left(A_{1}, \ldots, A_{n}\right)=\left(\widetilde{B}_{1}, \ldots, \widetilde{B}_{n}\right) \in\left(\mathbb{C}^{m \times m}\right)^{n}
$$

In [27] the authors were interested whether middle convolution preserves Schlesinger's deformation equations (5) for non-resonant Fuchsian systems and gave an affirmative answer to this question. In particular, the following statement holds.

Theorem 3 ([27]). Assume that there is no integer differences between any two distinct eigenvalues of $A_{j}$ and the Jordan canonical form of $A_{j}$ is independent of $a_{1}, a_{2}, \ldots, a_{n}$ for $j=$ $1,2, \ldots, n+1$. Then the Hitchin systems for the Fuchsian systems obtained by addition and middle convolution with parameters independent of $a_{1}, a_{2}, \ldots, a_{n}$ coincide with the Hitchin system for the initial Fuchsian system (8).

Note that Theorem 3 cannot be applied to a system with a resonant Fuchsian singular point, so it cannot be applied to non-Schlesinger isomonodromic deformations. 


\section{Non-Schlesinger isomonodromic deformations and middle convolution for resonant Fuchsian systems}

Since middle convolution preserves Schlesinger isomonodromic deformation equations [27], it is natural to ask what happens to non-Schlesinger isomonodromic deformations under middle convolution. In this paper we construct an explicit example to show that middle convolution does not in general preserve non-Schlesinger isomonodromic deformations. Our examples are based on the modifications of the Bolibruch example. Note that it is very important and of interest to specialists in the deformation theory to find new explicit examples of non-Schlesinger isomonodromic deformations because of the difficulty to write down the corresponding differential 1-form $\omega[4]$. Therefore, any new examples especially for Fuchsian systems of order higher than 2 are necessary. Our explicit examples show that under middle convolution the resonance condition may appear or disappear. Moreover, the maximal $i$-resonance of the system may change. This shows that in some cases the non-Schlesinger isomonodromic deformations are not preserved by middle convolution.

From non-Schlesinger to non-Schlesinger deformations. We shall present an example illustrating how to obtain new non-Schlesinger isomonodromic deformations by using middle convolution with changing the order of the resulting Fuchsian system. We remark that this example is non-trivial and new. We underline that the main difficulty with explicit examples of the non-Schlesinger deformations is to find the relevant differential 1-form.

In this illustrative example we prove that after applying middle convolution with a parameter $\mu, \mu \neq 0$, to a certain non-Schlesinger $(2 \times 2)$-isomonodromic family we get a new $(5 \times 5)$ family which is non-Schlesinger and it cannot be reduced to any Schlesinger isomonodromic deformation.

Consider a family of Fuchsian systems

$$
\begin{aligned}
\frac{d y}{d z}= & \left(\left(\begin{array}{cc}
\frac{1}{2} & 0 \\
-\frac{2 a}{a^{2}-1} & -\frac{1}{2}
\end{array}\right) \frac{1}{z+a}+\left(\begin{array}{cc}
0 & -6 a \\
0 & -\frac{1}{2}
\end{array}\right) \frac{1}{z}\right. \\
& \left.+\left(\begin{array}{cc}
1 & 3 a+3 \\
\frac{1}{1+a} & 3
\end{array}\right) \frac{1}{z-1}+\left(\begin{array}{cc}
-\frac{3}{2} & 3 a-3 \\
\frac{1}{a-1} & -2
\end{array}\right) \frac{1}{z+1}\right) y .
\end{aligned}
$$

This family has four singular points (since the residue matrix at infinity vanishes) with two resonant singularities: $a_{1}=-a$ with the maximal 1-resonance equal to 1 and $a_{3}=1$ with the maximal 3-resonance equal to 4 . The family is isomonodromic since the conditions of Theorem 1 are fulfilled with the differential form given by

$$
\begin{aligned}
\omega= & \left(\begin{array}{cc}
\frac{1}{2} & 0 \\
-\frac{2 a}{a^{2}-1} & -\frac{1}{2}
\end{array}\right) \frac{d(z+a)}{z+a}+\left(\begin{array}{cc}
0 & -6 a \\
0 & -\frac{1}{2}
\end{array}\right) \frac{d z}{z}+\left(\begin{array}{cc}
1 & 3 a+3 \\
\frac{1}{1+a} & 3
\end{array}\right) \frac{d(z-1)}{z-1} \\
& +\left(\begin{array}{cc}
-3 / 2 & 3 a-3 \\
\frac{1}{a-1} & -2
\end{array}\right) \frac{d(z+1)}{z+1}+\left(\begin{array}{cc}
0 & 0 \\
\frac{2 a}{a^{2}-1} & 0
\end{array}\right) \frac{d a}{z+a} .
\end{aligned}
$$

We remark that similarly to Bolibruch's example, the family (9) has an apparent singularity at $z=-a$ and can be transformed to a system with four singular points, including the point at infinity, and with residue matrices which do not depend on the parameter.

By applying middle convolution with the parameter $\mu\left(\mu \notin \frac{1}{2} \mathbb{Z}\right)$ to this family of Fuchsian systems and adding the vectors $e_{1}, e_{2}, e_{4}, e_{6}, e_{8}$ to the basis of the invariant subspaces we get a new $(5 \times 5)$-family. It has one resonant Fuchsian singularity $a_{1}=-a$ with the maximal 1 -resonance equal to 1 . We have

$$
\frac{d y}{d z}=\left(\frac{A_{1}(a)}{z+a}+\frac{A_{2}(a)}{z}+\frac{A_{3}(a)}{z-1}+\frac{A_{4}(a)}{z+1}\right) y
$$


where

$$
\begin{aligned}
& A_{1}(a)=\left(\begin{array}{ccccc}
\mu+1 / 2 & 0 & -6 a & 3 a+3 & 3 a-3 \\
-\frac{2 a}{a^{2}-1} & \mu-\frac{1}{2} & -\frac{1}{2} & 3 & -2 \\
0 & 0 & 0 & 0 & 0 \\
0 & 0 & 0 & 0 & 0 \\
0 & 0 & 0 & 0 & 0
\end{array}\right) \\
& A_{2}(a)=\left(\begin{array}{ccccc}
0 & 0 & 0 & 0 & 0 \\
0 & 0 & 0 & 0 & 0 \\
-\frac{2 a}{-1+a^{2}} & -\frac{1}{2} & \mu-\frac{1}{2} & 3 & -2 \\
0 & 0 & 0 & 0 & 0 \\
0 & 0 & 0 & 0 & 0
\end{array}\right) \text {, } \\
& A_{3}(a)=\left(\begin{array}{ccccc}
0 & 0 & 0 & 0 & 0 \\
0 & 0 & 0 & 0 & 0 \\
0 & 0 & 0 & 0 & 0 \\
-\frac{11 a+1}{6\left(a^{2}-1\right)} & -\frac{1}{2} & -\frac{5 a+1}{2(a+1)} & \mu+4 & -\frac{a+3}{a+1} \\
0 & 0 & 0 & 0 & 0
\end{array}\right) \text {, } \\
& A_{4}(a)=\left(\begin{array}{ccccc}
0 & 0 & 0 & 0 & 0 \\
0 & 0 & 0 & 0 & 0 \\
0 & 0 & 0 & 0 & 0 \\
0 & 0 & 0 & 0 & 0 \\
-\frac{9 a+1}{4\left(a^{2}-1\right)} & -\frac{1}{2} & \frac{5 a+1}{2(a-1)} & \frac{3(a-3)}{2(a-1)} & \mu-\frac{7}{2}
\end{array}\right) \text {. }
\end{aligned}
$$

This Fuchsian family with five singular points is isomonodromic since it is defined by the differential 1-form which satisfies conditions of Theorem 1. This form is given by

$$
\begin{aligned}
\omega= & A_{1}(a) \frac{d(z+a)}{z+a}+A_{2}(a) \frac{d z}{z}+A_{3}(a) \frac{d(z-1)}{z-1}+A_{4}(a) \frac{d(z+1)}{z+1} \\
& +\left(\begin{array}{ccccc}
0 & 0 & 6 & -3 & -3 \\
\frac{2\left(2 a^{2}+5 a+1\right)}{\left(a^{2}-1\right)^{2}} & \frac{2 a^{2}-5 a+1}{2\left(a^{2}-1\right) a} & -\frac{47 a^{2}+1}{2\left(a^{2}-1\right) a} & \frac{9 a+3}{a^{2}-1} & \frac{2(7 a+1)}{a^{2}-1} \\
0 & \frac{1}{2 a} & -\frac{1+5 a}{2 a-2 a^{3}} & 0 & 0 \\
-\frac{1}{6(1+a)^{2}} & \frac{1}{2(a+1)} & 0 & \frac{3}{a^{2}-1} & 0 \\
\frac{1}{4(a-1)^{2}} & \frac{1}{2(a-1)} & 0 & 0 & \frac{2}{a^{2}-1}
\end{array}\right) d a \\
& +\left(\begin{array}{ccccc}
0 & 0 & 0 & 0 & 0 \\
-\frac{2(2 \mu+1) a}{a^{2}-1} & 0 & \frac{24 a^{2}}{a^{2}-1} & -\frac{12 a}{a-1} & -\frac{12 a}{a-1} \\
0 & 0 & 0 & 0 & 0 \\
0 & 0 & 0 & 0 & 0 \\
0 & 0 & 0 & 0 & 0
\end{array}\right) \frac{d a}{z+a} .
\end{aligned}
$$

Moreover, the last term of the form $\psi(a) \frac{d a}{z+a}$ shows that this isomonodromic deformation is nonSchlesinger and cannot be reduced to any Schlesinger deformation. Also it is non-normalized because of the non-zero term of the form $\gamma(a) d a$.

We also remark that in general it is computationally difficult to find the non-Schlesinger isomonodromic differential 1-form for a given resonant Fuchsian family such that Theorem 1 is fulfilled. Actually, the form in our example is not unique, there exists a one-parameter family of such forms but we presented only the case when the parameter is zero. It is possible to simplify the calculations a bit by first deriving some identities from $d \omega=\omega \wedge \omega$, where $\omega$ is a 1-form with holomorphic coefficients, i.e., $\omega=A_{1}(a) d z /(z+a)+A_{2}(a) d z / z+A_{3}(a) d z /(z-$ 
1) $+A_{4}(a) d z /(z+1)+A_{5}(a) d a /(z+a)+B(a) d a$. For example, we have $\left[A_{1}, A_{5}\right]=A_{1}-A_{5}$, $\partial\left(A_{\infty}\right) / \partial a=-\left[A_{\infty}, B\right]$, where $A_{\infty}=-\left(A_{1}+A_{2}+A_{3}+A_{4}\right)$, and so on.

Example showing that non-Schlesinger deformations are not preserved by middle convolution. In this example we apply middle convolution with a parameter $\mu, \mu \neq 0$, to a certain non-Schlesinger isomonodromic $(2 \times 2)$-family (which is a modified Bolibruch example $(7)$ ) with five singular points (two of them are resonant). In the resulting family the resonances disappear and, hence, the resulting Fuchsian system cannot be included in any Bolibruch's nonSchlesinger isomonodromic deformation with a non-zero third term by Theorems 1 and 2 .

Consider a family

$$
\begin{aligned}
\frac{d y}{d z}= & \left(\left(\begin{array}{cc}
1 & 0 \\
-\frac{2 a}{a^{2}-1} & 0
\end{array}\right) \frac{1}{z+a}+\left(\begin{array}{cc}
0 & -6 a \\
0 & -1
\end{array}\right) \frac{1}{z}\right. \\
& \left.+\left(\begin{array}{cc}
\frac{3}{2}-\frac{\sqrt{21}}{2} & 3+3 a \\
\frac{1}{1+a} & -\frac{3}{2}-\frac{\sqrt{21}}{2}
\end{array}\right) \frac{1}{z-1}+\left(\begin{array}{cc}
-3 & 3 a-3 \\
\frac{1}{a-1} & 2
\end{array}\right) \frac{1}{z+1}\right) y
\end{aligned}
$$

with five singular points $-a, 0,1,-1, \infty$. The system has two resonant Fuchsian points $a_{1}=-a, a_{2}=0$ with the maximal resonances both equal to 1 . Note that this family is obtained by addition from Bolibruch's example. Recall that an addition for a Fuchsian system is a transformation of the form $y \rightarrow\left(z-a_{i}\right)^{-\alpha} y$ such that the corresponding to the singularity $a_{i}$ residue matrix $A_{i}$ is transformed by a shift, i.e., $A_{i} \rightarrow A_{i}+\alpha I_{p}$, where $I_{p}$ is an identity matrix of the same dimension as $A_{i}$. The residue matrix at infinity is the scalar. Therefore, it is straightforward to construct a corresponding 1-form. In particular, this family is isomonodromic since the conditions in Theorem 1 are fulfilled with the differential form given by

$$
\begin{aligned}
\omega= & \left(\begin{array}{cc}
1 & 0 \\
-\frac{2 a}{a^{2}-1} & 0
\end{array}\right) \frac{d(z+a)}{z+a}+\left(\begin{array}{cc}
0 & -6 a \\
0 & -1
\end{array}\right) \frac{d z}{z}+\left(\begin{array}{cc}
\frac{3}{2}-\frac{\sqrt{21}}{2} & 3+3 a \\
\frac{1}{1+a} & -\frac{3}{2}-\frac{\sqrt{21}}{2}
\end{array}\right) \frac{d(z-1)}{z-1} \\
& +\left(\begin{array}{cc}
-3 & 3 a-3 \\
\frac{1}{a-1} & 2
\end{array}\right) \frac{d(z+1)}{z+1}+\left(\begin{array}{cc}
0 & 0 \\
\frac{2 a}{a^{2}-1} & 0
\end{array}\right) \frac{d a}{z+a} .
\end{aligned}
$$

Applying middle convolution with the parameter $\mu=(1+\sqrt{21}) / 2$, which is chosen to be equal to the eigenvalue of the residue matrix at infinity, and adding vectors $e_{7}, e_{1}-e_{4}$ to the basis of the invariant subspaces, we get a new $(3 \times 3)$-family. The resulting family has no resonant singular points, so it cannot be an isomonodromic deformation with the differential form having the non-zero term of the form $\psi(a) \frac{d a}{z+a}$. One can also calculate that it is not a Schlesinger isomonodromic deformation. We were not able to find a flat differential 1-form $\omega=\frac{A_{1}(a) d(z+a)}{z+a}+\frac{A_{2}(a) d z}{z}+\frac{A_{3}(a) d z}{z-1}+\frac{A_{4}(a) d z}{z+1}+X(a) d a$ for a holomorphic $X$ to show that it is a non-Schlesinger isomonodromic deformation, which can be transformed to the Schlesinger one. Thus, middle convolution may destroy resonances in the residue matrices of the Fuchsian systems and, hence, non-Schlesinger deformations, which cannot be reduced to the Schlesinger ones. Explicitly, the resulting family of the Fuchsian systems is

$$
\frac{d y}{d z}=\left(\frac{A_{1}}{z+a}+\frac{A_{2}}{z}+\frac{A_{3}}{z-1}+\frac{A_{4}}{z+1}\right) y
$$

where

$$
\begin{aligned}
& A_{1}=\left(\begin{array}{ccc}
-6 a & -3 & \frac{1}{2}(12 a+\sqrt{21}+3) \\
0 & 0 & 0 \\
-6 a & -3 & \frac{1}{2}(12 a+\sqrt{21}+3)
\end{array}\right), \\
& A_{2}=\left(\begin{array}{ccc}
\frac{1}{2}(\sqrt{21}-1) & \frac{1}{a-1} & -\frac{2 a}{a^{2}-1}-\frac{\sqrt{21}}{2}+\frac{1}{2} \\
0 & 0 & 0 \\
0 & 0 & 0
\end{array}\right),
\end{aligned}
$$




$$
\begin{aligned}
& A_{3}=\left(\begin{array}{ccc}
\frac{1}{2}((9-\sqrt{21}) a-\sqrt{21}-3) & \frac{(\sqrt{21}+3)(a+1)}{2(a-1)}+3 & \alpha \\
\frac{1}{2}((9-\sqrt{21}) a-\sqrt{21}-3) & \frac{(\sqrt{21}+3)(a+1)}{2(a-1)}+3 & \alpha \\
\frac{1}{2}((9-\sqrt{21}) a-\sqrt{21}-3) & \frac{(\sqrt{21}+3)(a+1)}{2(a-1)}+3 & \alpha
\end{array}\right), \\
& \alpha=\frac{1}{2}(\sqrt{21}+3)(a+1)-6 a-1-\frac{(\sqrt{21}+3) a}{a-1}, \\
& A_{4}=\left(\begin{array}{ccc}
\frac{1}{2}((\sqrt{21}+3) a+\sqrt{21}+5) & -\frac{(\sqrt{21}+3)(a+1)+2}{2(a-1)} & -\frac{((\sqrt{21}+3) a+\sqrt{21}+5)\left(a^{2}-2 a-1\right)}{2\left(a^{2}-1\right)} \\
\frac{1}{2}((\sqrt{21}-9) a+\sqrt{21}+3) & -\frac{4 a+\sqrt{21}-1}{(a-1)} & -\frac{(\sqrt{21}-9) a^{2}-2(\sqrt{21}-2) a-\sqrt{21}-1}{2(a-1)} \\
\frac{1}{2}(\sqrt{21}+3)(a+1) & -\frac{(\sqrt{21}+3)(a+1)}{2(a-1)} & -\frac{(\sqrt{21}+3)\left(a^{2}-2 a-1\right)}{2(a-1)}
\end{array}\right) .
\end{aligned}
$$

It is interesting to note that family (11) can be transformed to a resonant one by a series of special transformations. The eigenvalues of the matrix $A_{1}$ are $\mu+1,0,0$, where $\mu$ is the parameter of middle convolution defined above, for $A_{2}$ the eigenvalues are $\mu-1,0,0$ and the matrix at infinity is diagonal with all diagonal elements equal to $\mu$. If in family (11) we first shift the residue matrix $A_{1} \rightarrow A_{1}-\mu I_{3}$ and then in the resulting family replace

$$
y=\left(\begin{array}{ccc}
(z+a) & f_{1}(a) & f_{2}(a) \\
0 & 1 & \frac{f_{2}(a)-1}{f_{1}(a)} \\
0 & 0 & 1
\end{array}\right) y_{1},
$$

where $f_{1}(a)$ and $f_{2}(a)$ are arbitrary holomorphic functions, we get a new family for $y_{1}$ with a resonant Fuchsian singularity at $z=-a$ and a resonant Fuchsian singularity at infinity. Moreover, applying the transformation $y_{1}=\operatorname{diag}(1 / z, 1,1) y_{2}$, we can get rid of the singularity at infinity. The resulting Fuchsian system remains resonant. See also [42] for other special transformations of Fuchsian systems.

\section{Discussion and open problems}

In this paper we have shown that middle convolution is useful in constructing new explicit examples of non-Schlesinger isomonodromic deformations for resonant Fuchsian systems. We have also shown that after middle convolution non-Schlesinger isomonodromic deformations may stay (see example (9)) or not (see example (10)). It is a new fact about middle convolution.

There are many open questions and problems in the theory of resonant Fuchsian systems and in understanding and explaining the results of middle convolution for them. They include:

1. The description of sufficient conditions when non-Schlesinger isomonodromic deformations of a Fuchsian system with a resonant singularity stay after middle convolution.

2. The description of sufficient conditions of the existence of non-Schlesinger isomonodromic deformations which cannot be reduced to the Schlesinger ones.

3. What kind of isomonodromic differential 1-form is obtained after middle convolution in general? How to reduce the complexity of straightforward calculations of the isomonodromic differential 1-form?

4. Is there any analogue of the Hitchin systems for resonant Fuchsian systems which will be invariant under middle convolution?

5. The construction of more examples of non-Schlesinger isomonodromic deformations (with or without the application of middle convolution, with and without apparent singularities). In particular, to our knowledge, there are no explicit examples of non-Schlesinger isomonodromic deformations with the order of poles greater then 1 in the "non-Schlesinger" term of the isomonodromic form. 
6. The (practical) description how the maximal resonance changes after middle convolution, including the cases when the resulting system is non-resonant.

Non-Schlesinger isomonodromic deformations have a natural analogue in theory of meromorphic systems with irregular singularities [5]. As mentioned in the introduction, there also exist generalizations of middle convolution to irregular systems (e.g., [28, 35, 49]). Therefore, almost all questions and problems mentioned above can be formulated for irregular systems as well. In particular, it is desirable to understand middle convolution for both resonant and nonresonant systems with irregular singularities and, in particular, to find more explicit examples of non-Schlesinger isomonodromic deformations of meromorphic systems with irregular resonant singularities.

\section{Acknowledgments}

Part of this work was carried out while Yu. Bibilo was visiting the Univerity of Warsaw in April 2014. The authors acknowledge the support of the Polish NCN Grant 2011/03/B/ST1/00330. Yu. Bibilo also acknowledges the support of the Russian Foundation for Basic Research (grant no. RFBR 14-01-00346 A).

\section{References}

[1] Anosov D.V., Concerning the definition of isomonodromic deformation of Fuchsian systems, Ulmer Seminaire über Funktionalysis und Differentialgleichungen (1997), no. 2, 1-12.

[2] Arinkin D., Fourier transform and middle convolution for irregular D-modules, arXiv:0808.0699.

[3] Arnold V.I., Arnold's problems, Springer-Verlag, Berlin, PHASIS, Moscow, 2004.

[4] Bibilo Yu., Inverse monodromy problems for systems with irregular singularities, Ph.D. Thesis, Moscow State University, 2012.

[5] Bibilo Yu., Isomonodromic deformations of systems of linear differential equations with irregular singularities, Sb. Math. 203 (2012), 826-843.

[6] Bolibruch A.A., On isomonodromic deformations of Fuchsian systems, J. Dynam. Control Systems 3 (1997), 589-604.

[7] Bolibrukh A.A., On isomonodromic confluences of Fuchsian singularities, Proc. Steklov Inst. Math. 221 (1998), 117-132.

[8] Bolibruch A.A., Inverse problems for linear differential equations with meromorphic coefficients, in Isomonodromic Deformations and Applications in Physics (Montréal, QC, 2000), CRM Proc. Lecture Notes, Vol. 31, Amer. Math. Soc., Providence, RI, 2002, 3-25.

[9] Bolibrukh A.A., Inverse monodromy problems in the analytic theory of differential equations, MCCME, Moscow, 2009.

[10] Bolibrukh A.A., Differential equations with meromorphic coefficients, Proc. Steklov Inst. Math. 272 (2011), $13-43$.

[11] Crawley-Boevey W., Shaw P., Multiplicative preprojective algebras, middle convolution and the DeligneSimpson problem, Adv. Math. 201 (2006), 180-208, math.RA/0404186.

[12] da Silva Jr. G., Kerr M., Pearlstein G., Arithmetic of degenerating principal variations of Hodge structure: examples arising from mirror symmetry and middle convolution, arXiv:1407.4102.

[13] Dettweiler M., Construction of relative motives with interesting étale realization using the middle convolution, in Mathematisches Institut, Georg-August-Universität Göttingen: Seminars Summer Term 2004, Universitätsdrucke Göttingen, Göttingen, 2004, 1-7.

[14] Dettweiler M., Middle convolution and Galois realizations, in Galois Theory and Modular Forms, Dev. Math., Vol. 11, Kluwer Acad. Publ., Boston, MA, 2004, 143-158.

[15] Dettweiler M., Reiter S., An algorithm of Katz and its application to the inverse Galois problem, J. Symbolic Comput. 30 (2000), 761-798. 
[16] Dettweiler M., Reiter S., Middle convolution of Fuchsian systems and the construction of rigid differential systems, J. Algebra 318 (2007), 1-24.

[17] Dettweiler M., Reiter S., Painlevé equations and the middle convolution, Adv. Geom. 7 (2007), 317-330, math.AG/0605384.

[18] Dettweiler M., Sabbah C., Hodge theory of the middle convolution, Publ. Res. Inst. Math. Sci. 49 (2013), 761-800, arXiv:1209.4185.

[19] Filipuk G., On the middle convolution and birational symmetries of the sixth Painlevé equation, $K u$ mamoto J. Math. 19 (2006), 15-23.

[20] Filipuk G.V., A hypergeometric system of the Heun equation and middle convolution, J. Phys. A: Math. Theor. 42 (2009), 175208, 11 pages.

[21] Filipuk G.V., Middle convolution and the hypergeometric equation, J. Phys. A: Math. Theor. 43 (2010), 175204, 10 pages.

[22] Flaschka H., Newell A.C., Monodromy- and spectrum-preserving deformations. I, Comm. Math. Phys. 76 (1980), 65-116.

[23] Fuchs R., Über lineare homogene Differentialgleichungen zweiter Ordnung mit drei im Endlichen gelegenen wesentlich singulären Stellen, Math. Ann. 63 (1907), 301-321.

[24] Garnier R., Sur des équations différentielles du troisième ordre dont l'intégrale générale est uniforme et sur une classe d'équations nouvelles d'ordre supérieur dont l'intégrale générale a ses points critiques fixes, Ann. Sci. École Norm. Sup. 29 (1912), 1-126.

[25] Garnier R., Étude de l'intégrale générale de l'équation VI de M. Painlevé dans le voisinage de ses singularités transcendantes, Ann. Sci. École Norm. Sup. 34 (1917), 239-353.

[26] Haraoka Y., Middle convolution for completely integrable systems with logarithmic singularities along hyperplane arrangements, in Arrangements of Hyperplanes - Sapporo 2009, Adv. Stud. Pure Math., Vol. 62, Math. Soc. Japan, Tokyo, 2012, 109-136.

[27] Haraoka Y., Filipuk G., Middle convolution and deformation for Fuchsian systems, J. Lond. Math. Soc. 76 (2007), 438-450.

[28] Hiroe K., Twisted Euler transform of differential equations with an irregular singular point, arXiv:0912.5124.

[29] Hitchin N., Geometrical aspects of Schlesinger's equation, J. Geom. Phys. 23 (1997), 287-300.

[30] Ilyashenko Y., Yakovenko S., Lectures on analytic differential equations, Graduate Studies in Mathematics, Vol. 86, Amer. Math. Soc., Providence, RI, 2008.

[31] Iwasaki K., Kimura H., Shimomura S., Yoshida M., From Gauss to Painlevé. A modern theory of special functions, Aspects of Mathematics, Vol. E16, Friedr. Vieweg \& Sohn, Braunschweig, 1991.

[32] Jimbo M., Miwa T., Monodromy preserving deformation of linear ordinary differential equations with rational coefficients. II, Phys. D 2 (1981), 407-448.

[33] Katsnelson V., Volok D., Deformations of Fuchsian systems of linear differential equations and the Schlesinger system, Math. Phys. Anal. Geom. 9 (2006), 135-186, math.CA/0506328.

[34] Katz N.M., Rigid local systems, Annals of Mathematics Studies, Vol. 139, Princeton University Press, Princeton, NJ, 1996.

[35] Kawakami H., Generalized Okubo systems and the middle convolution, Int. Math. Res. Not. 2010 (2010), $3394-3421$.

[36] Kitaev A.V., Non-Schlesinger deformations of ordinary differential equations with rational coefficients, J. Phys. A: Math. Gen. 34 (2001), 2259-2272, nlin.SI/0102019.

[37] Malgrange B., Sur les déformations isomonodromiques. I. Singularités régulières, in Mathematics and Physics (Paris, 1979/1982), Progr. Math., Vol. 37, Birkhäuser Boston, Boston, MA, 1983, 401-426.

[38] Mitschi C., Singer M.F., Projective isomonodromy and Galois groups, Proc. Amer. Math. Soc. 141 (2013), 605-617, arXiv:1002.2005.

[39] Oshima T., Katz's middle convolution and Yokoyama's extending operation, arXiv:0812.1135.

[40] Oshima T., Fractional calculus of Weyl algebra and Fuchsian differential equations, MSJ Memoirs, Vol. 28, Mathematical Society of Japan, Tokyo, 2012, arXiv:1102.2792.

[41] Patrikis S., Generalized Kuga-Satake theory and rigid local systems, I: the middle convolution, arXiv:1407.1942. 
[42] Poberezhnyi V.A., The general linear problem of the isomonodromic deformation of Fuchsian systems, Math. Notes 81 (2007), 529-542.

[43] Reiter S., Halphen's transform and middle convolution, arXiv:0903.3654.

[44] Schlesinger L., Über die Lösungen gewisser linearer Differentialgleichungen als Funktionen der singulären Punkte, J. Reine Angew. Math. 129 (1905), 287-294.

[45] Schlesinger L., Über eine Klasse von Differentialsystemen beliebliger Ordnung mit festen kritischen Punkten, J. Reine Angew. Math. 141 (1912), 96-145.

[46] Sibuya Y., Stokes phenomena, Bull. Amer. Math. Soc. 83 (1977), 1075-1077.

[47] Simpson C., Katz's middle convolution algorithm, Pure Appl. Math. Q. 5 (2009), 781-852, math.AG/0610526.

[48] Takemura K., Middle convolution and Heun's equation, SIGMA 5 (2009), 040, 22 pages, arXiv:0810.3112.

[49] Takemura K., Introduction to middle convolution for differential equations with irregular singularities, in New Trends in Quantum Integrable Systems, World Sci. Publ., Hackensack, NJ, 2011, 393-420, arXiv:1002.2535.

[50] Yamakawa D., Middle convolution and Harnad duality, Math. Ann. 349 (2011), 215-262, arXiv:0911.3863.

[51] Yamakawa D., Fourier-Laplace transform and isomonodromic deformations, arXiv:1306.0444. 